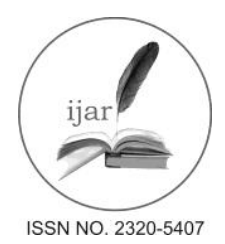

Journal homepage: http://www.journalijar.com
Journal DOI: 10.21474/IJAR01

\section{RESEARCH ARTICLE}

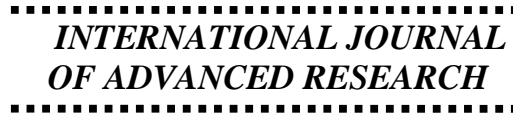

-

\title{
Long-Term Wind Speed Forecasting using Spiking Neural Network Optimized by Improved Modified Grey
} Wolf Optimization Algorithm.

*M. Madhiarasan ${ }^{1}$, and S. N. Deepa ${ }^{2}$.

1. Research Scholar (Ph.D), Department of Electrical and Electronics Engineering, Anna University Regional Campus, Coimbatore, Coimbatore - 641046, Tamil Nadu, India.

2. Associate Professor, Department of Electrical and Electronics Engineering, Anna University Regional Campus, Coimbatore, Coimbatore - 641046, Tamil Nadu, India.

\section{Manuscript Info}

\section{Manuscript History:}

Received: 18 May 2016

Final Accepted: 29 June 2016

Published Online: July 2016

Key words:

Spiking Neural Network,

Improved Spike Prop Algorithm,

Improved Modified Grey Wolf

Optimization Algorithm,

Hybrid Techniques,

Long-Term Forecasting,

Wind

*Corresponding Author

M. Madhiarasan.

\begin{abstract}
Wind speed forecasting is most needed due to its essentiality in wind farm and power system control and planning operation. Due to the increase of energy demands in order to meet the energy requirement wind energy receive a center of attraction because of its huge amount of availability and ecofriendly characteristics. Though numerous researches implemented different wind speed forecasting models, exact forecasting with the greatest accuracy is still thrusting topic in research. This article proposes two fold novel techniques for long-term wind speed forecasting namely improved spike prop algorithm incorporate spiking neural network (SNN) and improved modified grey wolf optimization algorithm (IMGWOA) based hybrid technique (SNN-IMGWOA). Proposed long-term forecasting technique using spiking neural network optimized through improved modified grew wolf optimization algorithm suitability and performance evaluation analyzed and compared with various earlier optimization algorithm namely GA, ES, PSO, ABC, GSA, CS, CSS and GWO and improved spike prop algorithm associated spiking neural network superiority confirmed with comparison between various traditional techniques such as Persistence, ARIMA, BPN, MLPN, RBFN, ELMAN Network and SVM. Simulations performed based on the observed real-time wind data's; numerical results and analyzes prove the virtue of proposed techniques.
\end{abstract}

Copy Right, IJAR, 2016,. All rights reserved.

\section{Introduction:-}

Wind speed forecasting is generally divided into four types' namely very short-term, short-term, medium-term and long-term wind speed forecasting. Long-term wind speed forecasting is doing favors in decision-making for reserve requirement, scheduling, dispatching, maintenance, integration, planning and operational management to aid optimal operating cost. Wind is complex and exhibits several levels of seasonality. Wind speed forecasting is very difficult due to the various factors influencing the wind speed and it is one of the important hot topics and this reflected in literature: Mohandes et al (2004) proposed wind speed prediction model based on support vector machine. Thanasis G. Barbounis et al (2006) presented local recurrent neural network based long-term forecasting model in order to forecasts wind speed and wind power. Tarek H. M. El-Fouly et al (2008) suggested linear time-series based prediction model for 24 hours ahead wind speed and direction prediction. Erasmo Cadenas and Wilfrido Rivera (2010) carried out work on hybrid (ARIMA-ANN) based forecasting model for wind speed forecasting. Gong Li and Jing Shi (2010) performed an analysis of one hour ahead wind speed forecasting using three artificial neural networks such as back propagation network, radial basis function network and adaptive linear element network. Hassen Bouzgou and Nabil Benoudjit (2011) carried out work on wind speed prediction using multiple architecture system (MAS). Ortiz-García et al (2011) performed banks of support vector machine based short-term wind speed 
prediction model. Akinci (2011) presented multi-layer feed-forward neural network based short-term wind speed forecasting. Aubai Alkhatib et al (2012) performed two layers of feed-forward back propagation with gradient algorithm based short-term wind speed prediction. Junghoon Lee et al (2012) pointed out artificial neural network based wind speed prediction. Gupta et al (2012) suggested a four layer back propagation neural network based wind speed forecasting in Jaipur. Wang et al (2012) presented radial basis function neural network based one hour ahead wind speed prediction.

Deligiorgi et al (2013) pointed out wind speed estimation by means of the artificial neural networks. Azad et al (2014) performed neural network based pattern recognition and long-term wind speed forecasting. Daraeepour and Echeverri (2014) proposed multi-variable neural network based day ahead hourly wind speed prediction. Diksha Kaur et al (2014) established hybrid approach (wavelet transform- Auto regressive moving average) based one hour ahead wind speed forecasting. Velo et al (2014) developed back propagation learning based multi-layer perceptron network for annual average wind speed estimation. Xinxinzhu et al (2014) suggested short-term wind speed forecasting using modified regime switching space-time diurnal method. Yin et al (2014) performed principal component analysis and rough set theory associated Elman neural network for short-term wind speed forecasting. Liu et al (2015) carried out a performance investigation of decomposing algorithms adopted multi-layer perceptron and ANFIS neural networks based hybrid wind speed forecasting models. Ramasamy et al (2015) presented daily mean wind speed prediction based on multi-layer perceptron network. Sangita B. Patil and Bapu K. Patil (2015) pointed out a day ahead wind speed prediction model using support vector machine. Wang et al (2015) suggested Elman recurrent neural network based hybrid forecasting models for medium-term wind speed forecasting. Madhiarasan and Deepa (2016) established wind speed forecasting using improved back propagation networks. Madhiarasan and Deepa (2016) developed different time scale wind speed prediction using novel ensemble neural networks and proposed approach performance substantiated with $\mathrm{K}$-fold cross validation. Madhiarasan and Deepa (2016) carried out comparative performance investigation of different time scale wind speed forecasting using six artificial neural networks. Remarks: Among the six artificial neural networks based forecasting model recursive radial basis function neural network perform well with the least statistical error and higher forecasting accuracy validation on all three wind farm data set.

Spiking neurons are an artificial neuron that resembles biological counterpart, a neural network developed based on these neurons called as spiking neural network. Hodgkin and Huxley (1952) pointed out spiking neural network (SNN) with the existence of several complexity implications. Gerstner (1995) evolved spike response model (SRM). Sander M Bohte et al (2001) developed supervised learning rule spike prop for spiking neural network. Yaochu Jin et al. (2007) carried out solution for classification issues by means of the spiking neural network with the introduction of pareto-based evolutionary optimization. Spiking neural network possesses special characteristics, namely enhanced network accuracy, more realistic and powerful computation compared with other traditional artificial neural networks.

Even though the artificial neural network has good ability to solve the complex problems in some cases it suffers from local minima, convergence issues, higher computational time and loss of generalization capability. Therefore, optimization algorithm introduced in artificial neural network to avoid the above said problems because optimization algorithm generally facilitates global optimal solution, faster convergence and assists the neural network to produce promising results with reduced computational time. Over past decades numerous optimization algorithms proposed based on inspiration of nature. Holland (1992) suggests a genetic algorithm (GA). Ingo Rechenberg (1994) pointed out evolution strategies (ES). Kennedy and Eberhart (1995) developed particle swarm optimization (PSO). Karaboga and Basturk (2007) established artificial bee colony (ABC) algorithm. Rashedi et al (2009) evolved gravitational search algorithm (GSA). Yang and Deb (2009) present cuckoo search (CS) algorithm. Kaveh and Talatahari (2010) performed charged system search (CSS) optimization algorithm. Mirjalili et al (2014) implements grey wolf optimizer (GWO). Madhiarasan and Deepa (2016) suggested Modified grey wolf optimizer (MGWO).

Henceforth, this article addresses new improved spike prop algorithm and novel hybrid technique (SNN-IMGWOA) applied for wind speed forecasting. Simulations performed based on the observed real-time wind data's. The proposed techniques have not been proposed by researcher elsewhere, it shows the novelty of this article.

This article organized as follows: Section 1 presents the introduction and literature review related to wind speed forecasting and spiking neural network. Section 2 illustrates about proposed improved spike prop associated spiking 
neural network framework and modeling. Proposed technique implementation process flow defined in Section 3. Section 4 describes the novel optimization algorithm and hybrid technique (SNN-IMGWOA). The simulation results of the proposed techniques and discussion are given in Section 5. Section 6 gives a conclusion.

\section{Proposed Technique Framework and Modeling:-}

Spiking neural network is one of the third generation artificial neural networks, which utilize the neural code of accurately timed spike. In spiking neural network information from one neuron conveyed to other neurons by means of spikes instead of analog signals. All neurons in a spiking neural networks communicated by means of spike. In spiking neural network inputs and output of spiking neurons called as spike train. Spike train is nothing but it is a series of firing times. One firing time represents the instant the neuron has fired a pulse or spike. Spiking neurons perform as a leaky Integrator of the incoming spikes and its potential modeled by a dynamic variable. Input weights impact on post synaptic potential (PSP), the PSP is increments (rise) for the positive weights and decrements (decay) for the negative weights. The neuron fires a spike when the predefined threshold level is less than the integrated sum of the incoming spike. Spike has depended on the PSP. Spiking neural network consists of numerous number of connections between independent neurons of successive layers. Spike response model (SRM) is a relationship between the input spikes and the internal state variables. Set of spikes with firing times $\mathrm{ft}$ applied to the input neurons $\mathrm{U}$ and hidden neurons $H$. When the membrane potential greater than the threshold value, the output neuron $\mathrm{O}$ fires a spike. Connection weights between the two neurons expressed as internal state variable. Figure.1 represents the architecture of the proposed perceptron spiking neural network, input layer composed of seven attributes, hidden layer contain nine hidden neurons and output layer consist of single neurons. Composition of several delayed synaptic terminals exist between each and every input neuron to hidden neuron and hidden neuron to output neuron. Figure. 2 depicts the linkage from hidden to the output neuron composed of several delays and synaptic terminals.

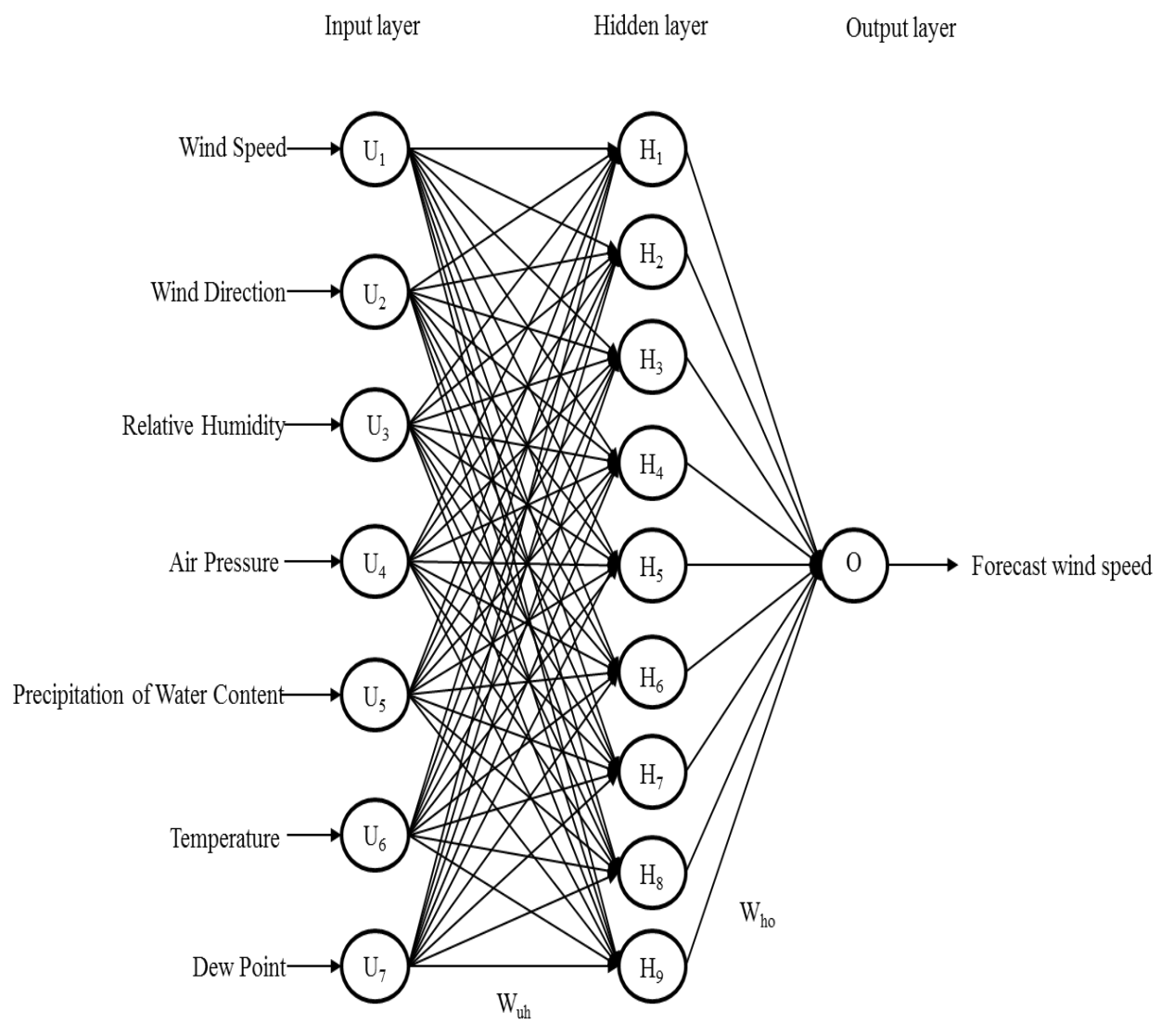

Figure.1:- Proposed perceptron spiking neural network architecture. 


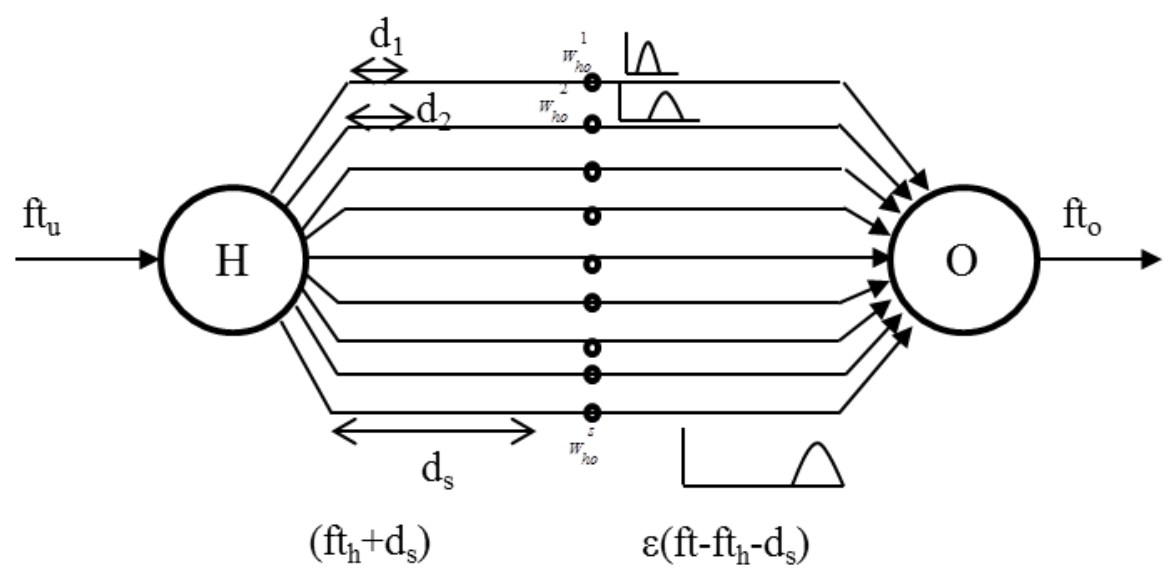

Figure.2:- Linkage from hidden to the output neuron composed of several delays and synaptic terminals.

\section{Mathematical Modeling:-}

The Improved spike prop algorithm proposed, the improved spike prop algorithm is a back propagation algorithm with incorporation of momentum factor. Improved spike prop algorithm formidable to make the faster convergence than the spike prop algorithm. Proposed improved spike prop algorithm performs the following computations.

Calculate un weighted contribution of input and hidden layer associated spikes to the synaptic terminals as given below:

$Z_{u}^{s}(f t)=\varepsilon\left(f t-f t_{u}-d_{s}\right)$

$Z_{h}^{s}(f t)=\varepsilon\left(f t-f t_{h}-d_{s}\right)$

$\varepsilon(f t)=f t / \tau_{m}\left(e^{1-f t / \tau_{m}}\right)$

$Z(f t)$ - un weighted contribution of all spikes to the synaptic terminals.

$d_{s}$ - Delay between the two nodes in the $s^{\text {th }}$ synaptic terminals.

$\varepsilon(f t)$ - Spike response function. It helps to shape the post synaptic potential (PSP) a neuron.

$\tau_{m}$ - Membrane potential decay (decrements) time constant.

Linkage weights between two neurons expressed as internal state variables. The internal state variables illustrated by means of spike response function, weighted by the synaptic efficacy.

Compute internal state vector of hidden neurons

$I_{h}(f t)=\sum_{u \in \Gamma^{h}} \sum_{s=1}^{k} W_{u h}^{s} Z_{u}^{s}(f t)$

Compute internal state vector of output neurons

$I_{o}(f t)=\sum_{h \in \Gamma_{h}} \sum_{s=1}^{k} W_{h o}^{s} Z_{h}^{s}(f t)$

Compute statistical error

$e=1 / 2 * \sum\left(f t_{o}^{\text {real }}-f t_{o}^{\text {expected }}\right)^{2}$

Weights modification,

Weight adjustment between hidden to output layer 


$$
\begin{aligned}
& \Delta W_{h o}^{s}=-\alpha Z_{h}^{s}\left(f t_{o}^{\text {real }}\right) \cdot \delta_{o} \\
& \delta_{o}=\frac{\left(f t_{o}^{\text {expected }}-f t_{o}^{\text {real }}\right)}{\sum_{h \in \Gamma_{h}} \sum_{s=1}^{k} W_{h o}^{s}\left(\frac{\partial Z_{h}^{s}\left(f t_{o}^{\text {real }}\right)}{\partial f t_{o}^{\text {real }}}\right)}
\end{aligned}
$$

Weight adjustment between inputs to hidden layer

$$
\begin{aligned}
& \Delta W_{u h}^{s}=-\alpha Z_{u}^{s}\left(f t_{h}^{\text {real }}\right) \cdot \delta_{h} \\
& \delta_{h}=\frac{\sum_{o \in \Gamma^{h}} \delta_{o}\left\{\sum_{s=1}^{k} W_{h o}^{s}\left(\frac{\partial Z_{h}^{s}\left(f t_{o}^{\text {real }}\right)}{\partial f t_{o}^{\text {real }}}\right)\right\}}{\sum_{u \in \Gamma^{h}} \sum_{s=1}^{k} W_{u h}^{s}\left(\frac{\partial Z_{u}^{s}\left(f t_{h}^{\text {real }}\right)}{\partial f t_{h}^{\text {real }}}\right)}
\end{aligned}
$$

Weight updating with the inclusion of momentum factor

$$
\begin{aligned}
& W_{u h}(i+1)=W_{u h}(i)+\Delta W_{u h}^{s}+\eta\left(W_{u h}(i)-W_{u h}(i-1)\right) \\
& W_{h o}(i+1)=W_{h o}(i)+\Delta W_{h o}^{s}+\eta\left(W_{h o}(i)-W_{h o}(i-1)\right)
\end{aligned}
$$

\section{Implementation Process Flow:-}

The proposed wind speed forecasting technique based on 3 layered spiking neural networks with a single hidden layer consist of nine hidden neurons step by step process flow is given as follows:

\section{Process 1: Data observation and data scaling}

Real-time data patterns consist of seven input variables, namely wind speed, wind direction, relative humidity, air pressure, precipitation of water content, temperature, dew point utilized in this research obtained from National Oceanic and Atmospheric Administration, United States over the period from January 2003 to December 2014. The observed wind data consists of 1,50,000 data patterns for each considered input variable. Observed real-time input variables minimum and maximum ranges presented in Table.1.

The Table. 1 shows that the observed data patterns are raw real-time data's consist of various units and various ranges. Hence, data scaling required scales the raw real-time data in the range of 0 to 1 . In this work min max data scaling method used and its computation equation is given as below:

Scaled real-time observed data's, $U_{o b}^{\prime}=\left(\frac{U_{o b}-U_{o b_{-} \min }}{U_{o b_{-} \max }-U_{o b_{-} \min }}\right)\left(U_{t_{-} \max }^{\prime}-U_{t_{-} \min }^{\prime}\right)+U_{t_{-} \text {min }}^{\prime}$

where, $U_{o b}$ - observed input data, $U_{o b_{-} \min }$ - minimum of observed input data, $U_{o b_{-} \max }$ - maximum of observed input data, $U_{t_{-} \min }^{\prime}$ - minimum of the target, $U_{t_{-} \max }^{\prime}$ - maximum of the target.

Table.1:- Real-time data patterns range.

\begin{tabular}{|c|c|c|c|}
\hline S. No & Input Variables & Units & Range of input variables \\
\hline 1 & Wind Speed & $m / s$ & $1-14$ \\
\hline 2 & Wind Direction & Degree & $0.1-360$ \\
\hline 3 & Relative Humidity & Percentage & $48-98$ \\
\hline 4 & Air Pressure & mbar & $1000-1030$ \\
\hline 5 & Precipitation of Water Content & Percentage & $15-60$ \\
\hline 6 & Temperature & Degree. Celsius & $17-32$ \\
\hline
\end{tabular}

Process 2: Initialize and set the implementation parameters, as given in Table.2. 
Table.2:- Spiking neural network implementation parameters

\begin{tabular}{|l|l|c|}
\hline Proposed Technique & Parameters & Parametric Values \\
\hline SNN & Input neurons & 7 \\
\hline & Number of hidden layer & 1 \\
\hline & Number of hidden neurons & 9 \\
\hline & Output neuron & 1 \\
\hline & Epoch & 600 \\
\hline & Delay time constant $\left(\tau_{\mathrm{m}}\right)$ & 0.0001 \\
\hline & Learning rate $(\alpha)$ & 0.9 \\
\hline & Momentum factor $(\eta)$ & $\mathrm{ms}$ \\
\hline
\end{tabular}

Process 3: Training and testing stages

In general, the performance of the neural network influence by means of the training and testing set division, proposed techniques tested with various divisions of training and testing set while the performance improves by $70 \%$ of data's for training and $30 \%$ of data's for testing. Hence, during training and testing stages, observed data patterns are randomly divided into training (70\% of data patterns) and testing (30\% data patterns) sets.

Process 4: Compute forecast performance indicator of trained neural network

Proposed technique, performances analyzed based on the mean square error (MSE), mean absolute error (MAE), root mean square error (RMSE) and mean absolute percentage error (MAPE) criteria. The forecast performance indicator equations are given as below:

$\operatorname{MSE}=\frac{1}{N} \sum_{d p=1}^{N}\left(O_{d p}^{\prime}-O_{d p}\right)^{2}$

$\mathrm{MAE}=\frac{1}{N} \sum_{d p=1}^{N}\left(O_{d p}^{\prime}-O_{d p}\right)$

$\operatorname{RMSE}=\sqrt{\frac{1}{N}} \sum_{d p=1}^{N}\left(O_{d p}^{\prime}-O_{d p}\right)^{2}$

MAPE $=\frac{100}{N} \sum_{d p=1}^{N}\left|\left(O_{d p}^{\prime}-O_{d p}\right) / \bar{O}_{d p}\right|$

where, $\mathrm{N}$ - number of data patterns, $O_{d p}^{\prime}$ - observed real output, $\bar{O}_{d p}$ - average observed real output, $O_{d p}$ is forecast output.

\section{Optimization Technique (IMGWOA):-}

In neural network weight optimization is an essential process and its leads to the best network accuracy. Therefore, this article proposes a new optimization algorithm, namely improved modified grey wolf optimization algorithm for spiking neural network in order to do weights and delays optimization. Improved modified grey wolf optimization algorithm explicitly overcomes the implications such as tendency to stick into local minima, higher computational time and slower convergence. Efficacy of IMGWOA analyzed for spiking neural network weights and delays optimization, application of wind speed forecasting.

\section{Improved Modified Grey Wolf Optimization Algorithm (IMGWOA)}

Mirjalili et al (2014) established GWO to obtain better performance by finding the global optimum. In this article improved modified grey wolf optimization algorithm proposed, which resembles the features of grey wolf hunting and social leadership. Grey wolf hunting involves the following steps: explore target to attack (prey or victim), chasing and around the target, exploit the target. In order to reduce computational complexity and convergence of the grey wolf optimizer, division of populations minimized as three groups instead of four groups because generally wolves' $\theta$ and $\zeta$ are the most responsible and important wolves which have the capability to guide other wolves. 
Hence, the subordinate wolves and the least rank wolves grouped in the single group which reduce the computational complexity and meanwhile the worst position of each group considered in the updating process which minimized convergence time and leads to improved performance. Improved Modified grey wolf optimization algorithm facilitates higher rank of exploration and exploitation compared with GWO and other existing algorithms. The population is split into three groups theta $(\theta)$, zeta $(\zeta)$ and psi $(\psi)$ for the proposed IMGWOA.

Promising area of the solution space achieved by the wolves $\psi$ based on the guidance of the first two fittest wolves such as theta $(\theta)$ and zeta $(\zeta)$. The wolves update their position around $\theta$ and $\zeta$ during optimization process are given as below:

$\vec{D}=\left|\vec{G} \cdot \vec{P}_{p}(i)-\vec{P}(i)-\vec{P}_{\text {worst }}(i)\right|$

$\vec{P}(i+1)=\vec{P}(i)-\vec{H} \cdot \vec{D}$

where, $i$ - Current Epoch, $\vec{P}$ - Position vector of the grey wolf, $\vec{P}_{p}$ - Position vector of the prey, $\vec{H} \& \vec{G}$ Coefficient vectors.

Coefficient vectors computed as follows:

$\vec{H}=2 \vec{u} \cdot \vec{v}_{1}-\vec{u}, \vec{G}=2 \cdot \vec{v}_{2}$ where, $\vec{u}$ - Set to linearly decreased from 2 to 0 during the course of epochs, $\vec{v}_{1} \&$ $\vec{v}_{2}$ - Random vector between $0 \& 1$.

The rest of the search agents ( $\psi$ wolves) update their position based on the best first two fitness solutions such as $\theta, \zeta$ expressed as follows:

$$
\begin{aligned}
& \vec{D}_{\theta}=\left|\vec{G}_{1} \cdot \vec{P}_{\theta}-\vec{P}-\vec{P}_{\text {worst }}\right| \\
& \vec{D}_{\zeta}=\left|\vec{G}_{2} \cdot \vec{P}_{\zeta}-\vec{P}-\vec{P}_{\text {worst }}\right| \\
& \vec{P}_{1}=\vec{P}_{\theta}-\vec{H}_{1} \cdot\left(\vec{D}_{\theta}\right) \\
& \vec{P}_{2}=\vec{P}_{\zeta}-\vec{H}_{2} \cdot\left(\vec{D}_{\zeta}\right) \\
& \vec{P}(i+1)=\frac{\left(\vec{P}_{1}+\vec{P}_{2}\right)}{2}
\end{aligned}
$$

where, $\vec{P}_{\theta}$ - Position of the first best solution $(\theta), \vec{P}_{\zeta}$ - Position of the second best solution $(\zeta), \vec{P}$ - Current position, $i$ - Number of epochs, $\vec{H}_{1}, \vec{H}_{2}$ - Random vectors.

The approximate distance between the present solution and $\theta, \zeta$ respectively computed based on the equations (20)-(21). The final position of the present solution computed after the distance computations are expressed in equations (22)-(24). Coefficient vectors are highly influenced for exploration and exploitation; if $G>1$ it promoted exploration meanwhile $|H|<1$ exploitation is emphases.

\section{SNN-IMGWOA:-}

Spiking neural network produce the least performance forecast indicator (MSE) with the best combination of inter linkage weights and delays, but exploring the best inter linkage weights and delays are tough task and become attractive in research fields. Hence, spiking neural network the best optimal inter linkage weights and delays obtained using a new Meta heuristic algorithm (IMGWOA). The presented hybrid technique (SNN-IMGWOA) based wind speed forecasting process flow depicted in Figure. 3 and block diagram representation of the proposed technique shown in Figure.4. Mean square error (MSE) is generally used performance measure for evaluation of a spiking neural network. Hence, the minimization of MSE is used as a fitness function for the proposed IMGWOA. The optimal weights and delays of the spiking neural network provided by means of the IMGWOA, IMGWOA perform minimization MSE based on modification of weights and delays. Weights and delays modifying of wolves are highly influenced based on the $\theta$ wolf, the best weights and delays searched by means of the IMGWOA in order to improve the convergence, accuracy and minimizes spiking neural network error. Weights and delays optimized 
until MSE reaches to negligible lower value. An improved Modified Gray Wolf Optimization algorithm (IMGWOA) posse better convergence and reduces computational complexity than that of the GWO. In order to substantiate the performance capability of the proposed technique (SNN-IMGWOA), spiking neural network weight optimization performed with the different existing optimization algorithms, namely GA, ES, PSO, ABC, GSA, CS, CSS and GWO.

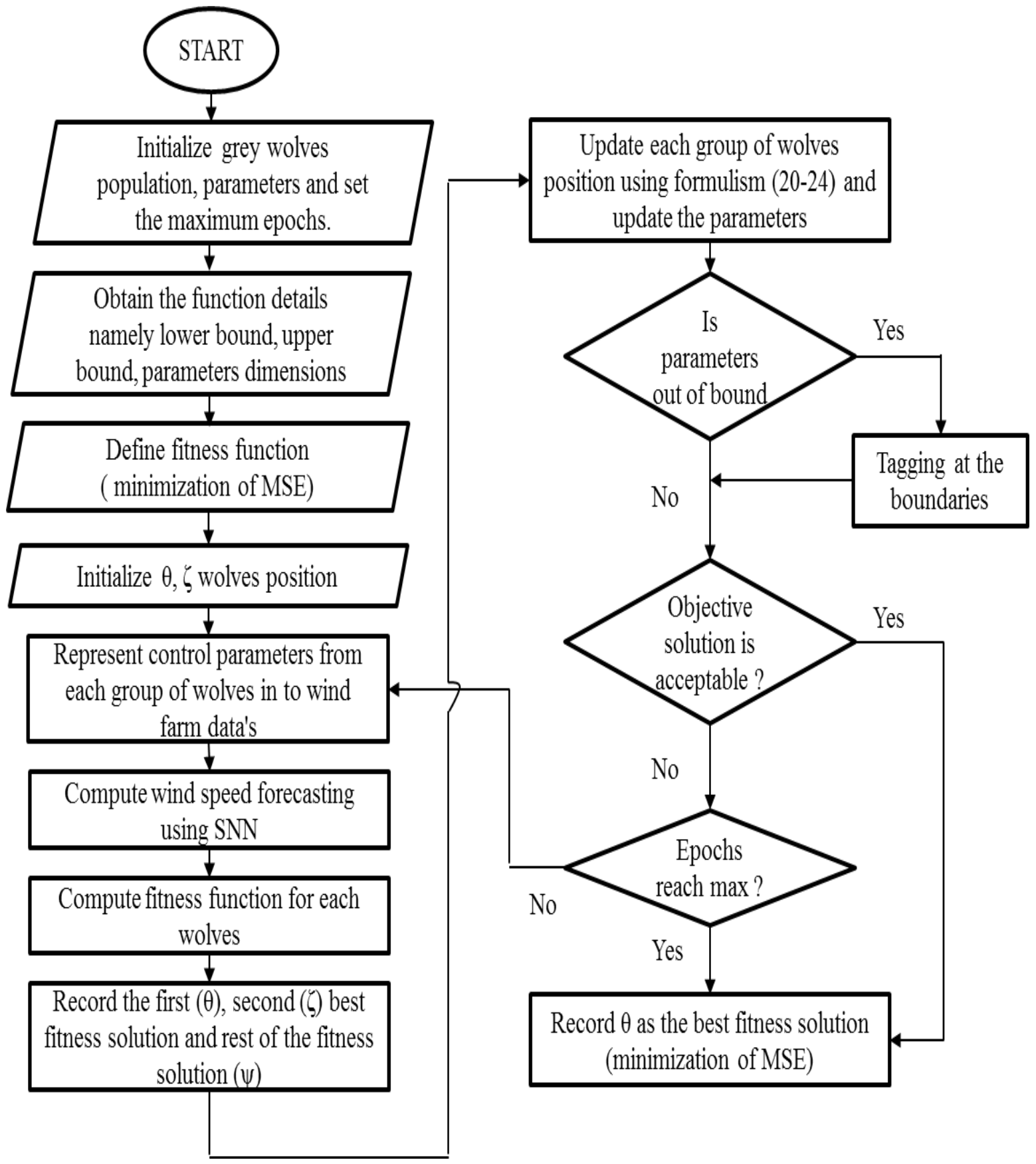

Figure.3:- Hybrid technique process flow. 


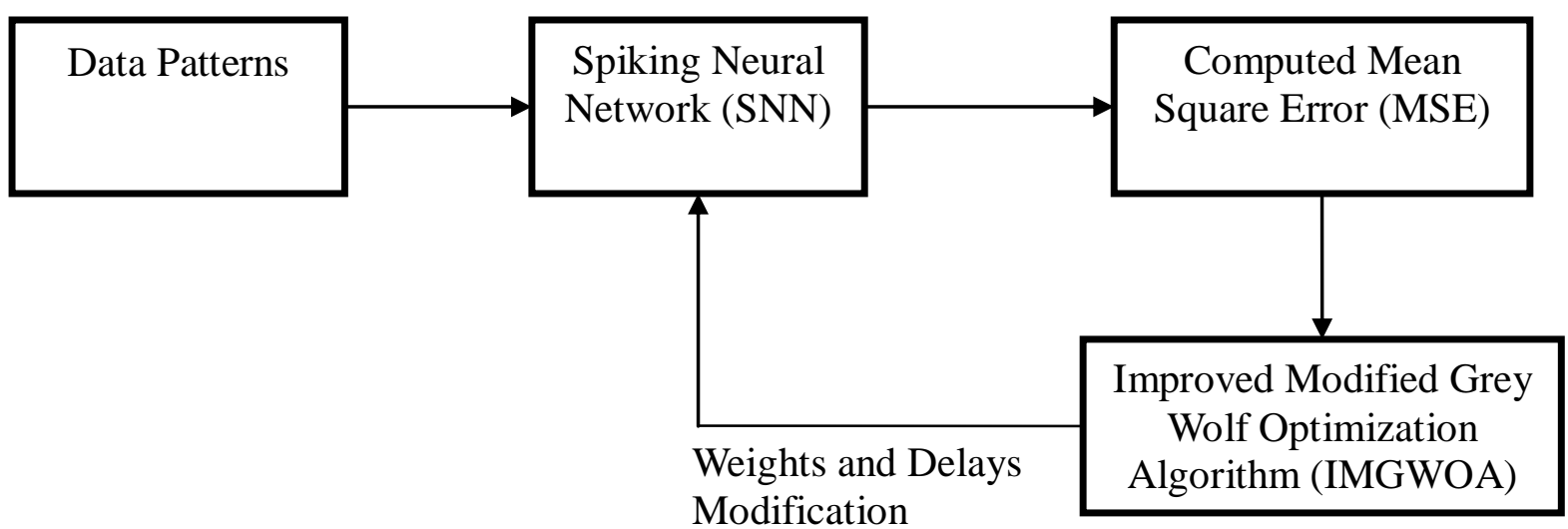

Figure.4:- Pictorial representation of SNN-IMGWOA.

\section{Results and discussion:-}

Simulation of proposed SNN and SNN-IMGWOA performed in MATLAB version 2009(a). The hardware configuration of the personal computer utilized is an Acer dual core processor with 2GHZ, 2 GB RAM and the operating system utilized is window 7.

\section{Forecasting result, comparative analysis with traditional methods:-}

Improved spike prop algorithm expedites speed up convergence by the inclusion of a momentum factor in back propagation stage. Compared with sigmoidal neurons, network with spiking neurons are computationally much stronger and facilitate enhanced accuracy. Numerical results illustrate the capability of the proposed spiking neural network with an improved spike prop algorithm for wind speed forecasting. Table. 3 compares the simulation results achieved by traditional techniques and proposed technique. From the Table. 3 it is obvious that the presented improved spike prop algorithm associated SNN based winds speed forecasting technique result with the least forecast performance indicators when compared to other methods. Simulation results achieved by the proposed technique such as a comparison between observed and forecast wind speed and the relation between observed and forecast wind speed shown in Figure.5 and 6. Due to the space limitation only a part of the result with 8000 data patterns represented in Figure.5. According to the Figure.5 it could be noticed that the forecast wind speeds are greatly matched with the observed wind speed for long-term horizon forecasting. Figure. 6 gives a clear view about the linear relationship between forecast and observed wind speed. Forecast performance indicator vs. Forecasting techniques, graph shown in Figure.7, from this figure, it could be seen that SNN based wind speed forecasting made the best performance in terms of very lower forecast performance indicators than other traditional techniques.

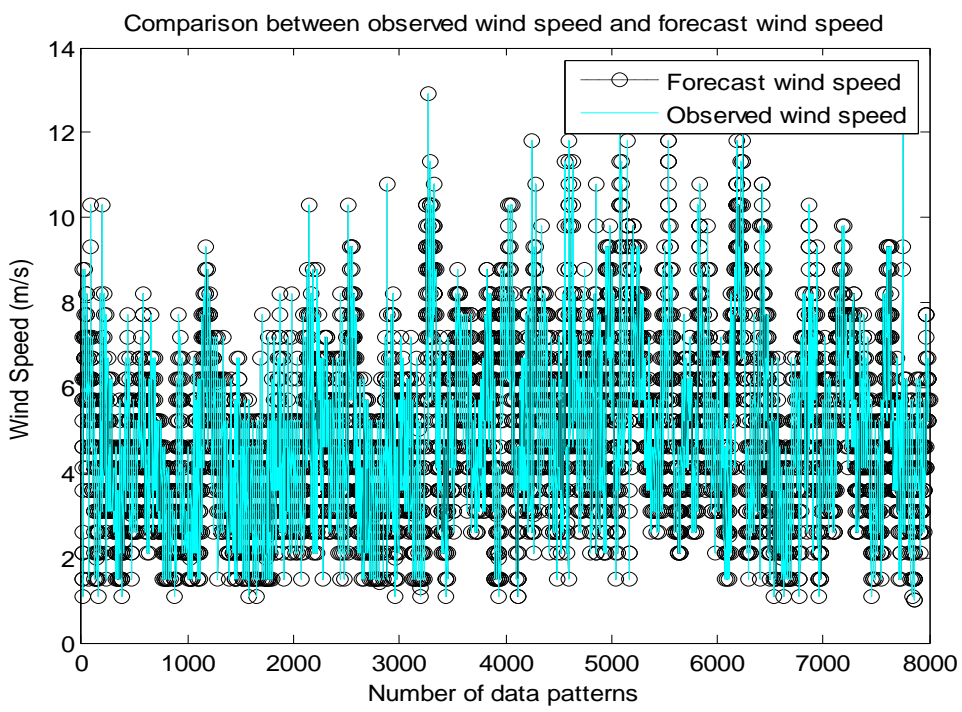

Figure.5:- Comparison between observed and forecast wind speed. 


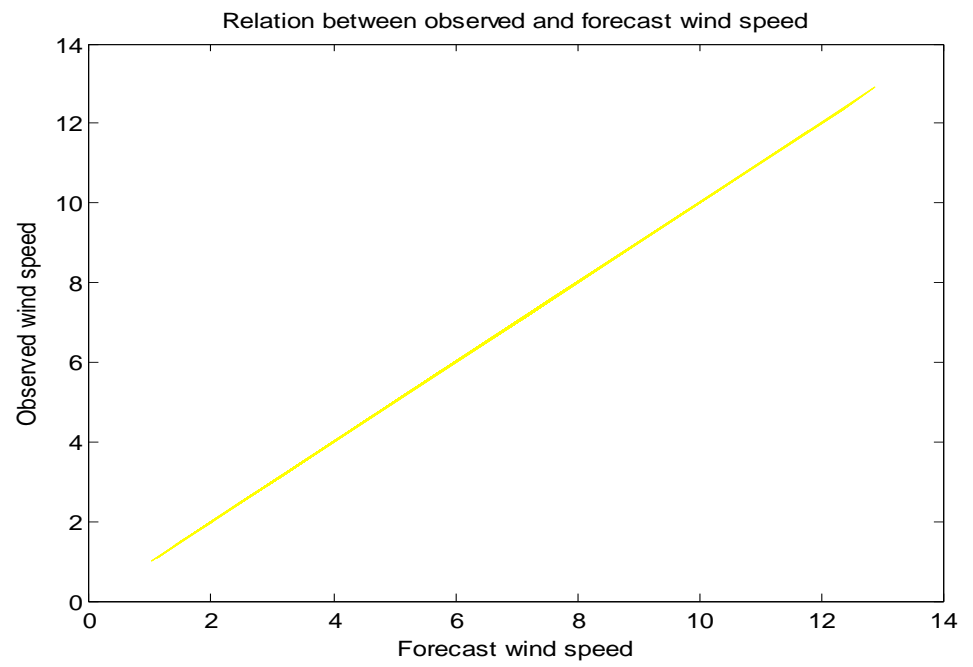

Figure.6:- Relation between observed and forecast wind speed.

Table.3:- Numerical results comparison between traditional and proposed techniques.

\begin{tabular}{|c|c|c|c|c|}
\hline \multirow{2}{*}{ Techniques } & \multicolumn{4}{|c|}{ Forecast Performance Indicator } \\
\cline { 2 - 5 } & MSE & MAE & RMSE & MAPE \\
\hline Persistent & 3.8425 & 1.3496 & 1.9602 & 39.3220 \\
\hline ARIMA & 0.0046 & 0.0302 & 0.0675 & 0.3725 \\
\hline BPN (7-9-1) & 0.0023 & 0.0088 & 0.0476 & 0.2573 \\
\hline MLPN (7-9-1) & $1.5528 \mathrm{e}-06$ & 0.0010 & 0.0012 & 0.0305 \\
\hline RBFN (7-9-1) & $6.7797 \mathrm{e}-07$ & $6.7235 \mathrm{e}-04$ & $8.2339 \mathrm{e}-04$ & 0.0196 \\
\hline EN (7-9-1) & 0.0025 & 0.0354 & 0.0503 & 0.4364 \\
\hline SVM (7-9-1) & $1.6021 \mathrm{e}-07$ & $3.4294 \mathrm{e}-04$ & $4.0026 \mathrm{e}-04$ & 0.0100 \\
\hline SNN (7-9-1) & $2.7362 \mathrm{e}-11$ & $3.4550 \mathrm{e}-06$ & $5.2309 \mathrm{e}-06$ & $7.1403 \mathrm{e}-05$ \\
\hline
\end{tabular}

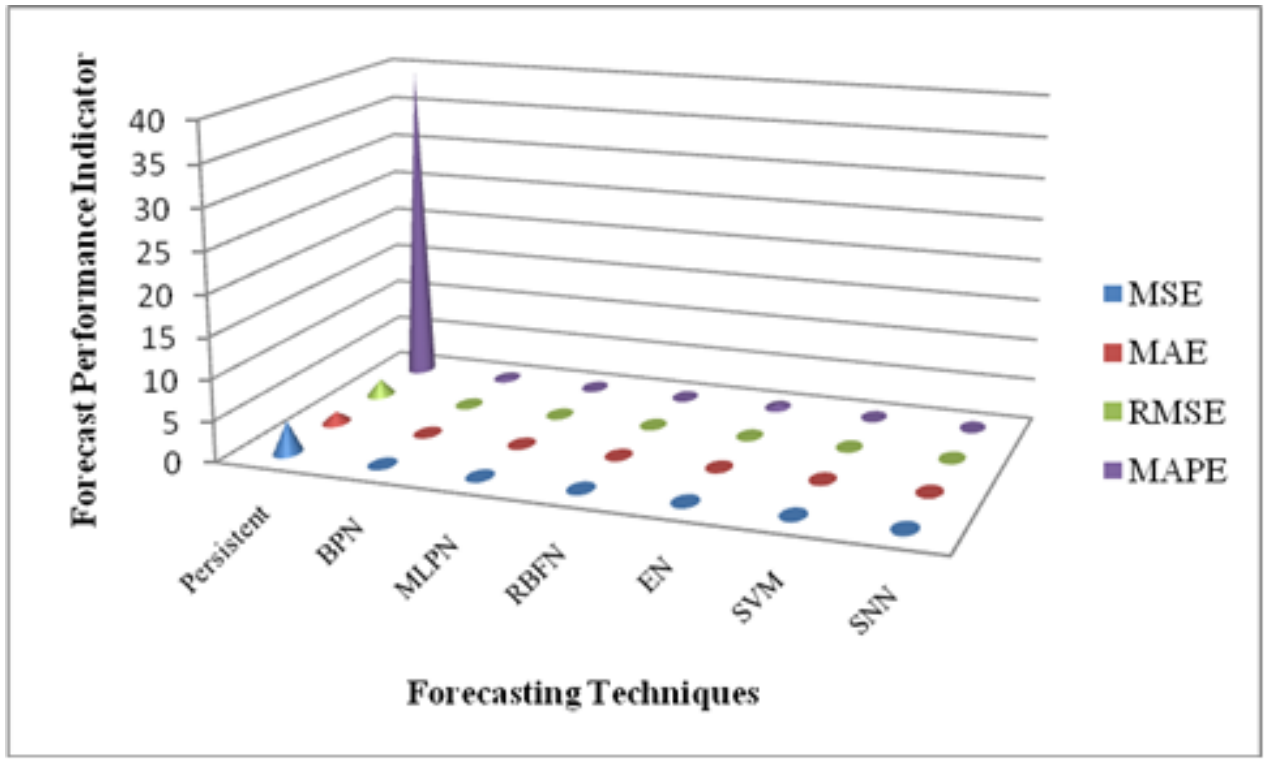

Figure.7:- Forecasting techniques vs. performance indicators. 
Forecasting results comparative analysis with other algorithms:-

The proposed long-term wind speed forecasting hybrid technique SNN-IMGWOA effectiveness verified by performing simulations on observed real-time wind data's and compared the results obtained with other earlier algorithms. The obtained results averaged over a 30 trials make sure network parameters fixed as constant for each run. On each trial optimization algorithm applied for a maximum of 200 epochs, population size is set as 100 and $\vec{u}$ is linearly decrements from 2 to 0 during the epoch. Quantitative and qualitative analyzes carried out for SNN weights and delays optimization with different existing and proposed optimization algorithm tabulated in Table.4. Numerical simulation results and analysis evidence that the developed SNN-IMGWOA based wind speed forecasting technique achieve extremely higher forecasting accuracy in terms of smaller performance error indicator (MSE) than that of other existing algorithms.

Table.4. Experimental comparison between earlier and proposed algorithm based SNN.

\begin{tabular}{|l|c|}
\hline \multicolumn{1}{|c|}{ Algorithms } & MSE AVG \pm STD \\
\hline SNN-GA & $2.8549 \mathrm{e}-16 \pm 8.1401 \mathrm{e}-09$ \\
\hline SNN-ES & $3.4205 \mathrm{e}-14 \pm 3.5879 \mathrm{e}-07$ \\
\hline SNN-PSO & $7.8323 \mathrm{e}-18 \pm 4.0248 \mathrm{e}-10$ \\
\hline SNN-ABC & $1.7235 \mathrm{e}-16 \pm 2.8920 \mathrm{e}-09$ \\
\hline SNN-GSA & $4.0771 \mathrm{e}-17 \pm 1.8663 \mathrm{e}-10$ \\
\hline SNN-CS & $1.7226 \mathrm{e}-18 \pm 6.8523 \mathrm{e}-10$ \\
\hline SNN-CSS & $4.2305 \mathrm{e}-19 \pm 3.5879 \mathrm{e}-11$ \\
\hline SNN-GWO & $8.3329 \mathrm{e}-20 \pm 1.6196 \mathrm{e}-12$ \\
\hline SNN-IMGWOA & $\mathbf{1 . 2 3 3 0 e - 2 4} \pm \mathbf{7 . 6 4 4 8 e}-19$ \\
\hline
\end{tabular}

\section{Conclusion:-}

In this article a novel (SNN-IMGWOA) hybrid based forecasting technique and improved spike prop algorithm adopted spiking neural network proposed for wind speed forecasting in the long-term forecast horizon. The suggested improved spike prop algorithm incorporated spiking neural network's outperform ability proven with a comparison of other traditional techniques such as persistence, ARIMA, BPN, MLP, RBFN, EN and SVM. In this work different earlier optimization algorithm, namely GA, ES, PSO, ABC, GSA, CS, CSS and GWO used for weights and delays optimization of spiking neural network due to explicitly prove that the proposed improved modified grey wolf optimization algorithm based spiking neural network make superior terrific efficiency. Comparative analysis implies that the proposed techniques make very encouraging higher forecasting accuracy with low forecast performance indicators.

\section{Acknowledgement:-}

The authors would like to express their thanks to National Oceanic and Atmospheric Administration, United States for providing the real-time wind farm data's. Mr. Madhiarasan, M was supported by the Rajiv Gandhi National Fellowship (F1-17.1/2015-16/RGNF-2015-17-SC-TAM-682 / (SA-III/Website)).

\section{Reference:-}

1. Akinci, T.Ç. (2011). Short Term Wind Speed Forecasting with ANN in Batman, Turkey. ELECTRONICS AND ELECTRICAL ENGINEERING / ELEKTRONIKA IR ELEKTROTECHNIKA. 107: 41-45.

2. Aubai Alkhatib., Siegfried Heier. and Melih Kurt. (2012). Detailed Analysis for Implementing a Short Term Wind Speed Prediction Tool Using Artificial Neural Networks. International Journal on Advances in Networks and Services. 5: 149-158.

3. Azad, H.B., Mekhilef, S. and Ganapathy, V.G. (2014). Long-term wind speed forecasting and general pattern recognition using neural networks. IEEE Transactions on Sustainable Energy. 5: 546-553.

4. Daraeepour, A. and Echeverri, D.P. (2014). Day-ahead wind speed prediction by a Neural Network-based model. 2014 IEEE Power and Energy Society Innovative Smart Grid Technologies Conference, Washington, DC, United States.

5. Deligiorgi, D., Philippopoulos, K. and Kouroupetroglou, G. (2013). Artificial neural network based methodologies for the estimation of wind speed. Green Energy and Technology. 129: 247-266.

6. Diksha Kaur., Tek Tjing Lie., Nirmal, K.C. Nair. and Brice Vallès. (2014). Wind Speed Forecasting Using Hybrid Wavelet Transform-ARMA Techniques. AIMS Energy. 3: 13-24. 
7. Erasmo Cadenas. and Wilfrido Rivera. (2010). Wind speed forecasting in three different regions of Mexico, using a hybrid ARIMA-ANN model. Renewable Energy. 35: 2732-2738.

8. Gerstner, W. (1995). Time structure of the activity in neural network models. Phys. Rev. E. 51: 738-758.

9. Gong Li. and Jing Shi. (2010). On comparing three artificial neural networks for wind speed forecasting. Applied Energy. 87: 2313-2320.

10. Gupta, R.A., Rajesh Kumar. and Ajay Kumar Bansal. (2012). Novel Topology for Wind Speed Forecasting based on Artificial Neural Network at Jaipur. Proc. of the Intl. Conf. on Advances in Computer, Electronics and Electrical Engineering: 333-338.

11. Hassen Bouzgou. and Nabil Benoudjit. (2011). Multiple architecture system for wind speed prediction. Applied Energy. 88: 2463-2471.

12. Holland, J.H. (1992). Genetic algorithms. Scientific American. 267: 66-72.

13. Hodgkin, A.L. and Huxley, A.F. (1952). A Quantitative Description of Ion Currents and its Applications to Conductance and Excitation in Nerve Membranes. Journal of Physiology (London). 117: 500-544.

14. Ingo Rechenberg. (1994). Evolutions strategie '94. Stuttgart: Frommann-Holzboog 1994.

15. Junghoon Lee., Gyung-Leen Park. and Eel-Hwan Kim. (2012). Wind Speed Modeling based on Artificial Neural Networks for Jeju Area. International Journal of Control and Automation. 5: 81-88.

16. Karaboga, D. and Basturk, B. (2007). A powerful and efficient algorithm for numerical function optimization: artificial bee colony (ABC) algorithm. Journal of Global Optimization. 39: 459-471.

17. Kaveh, A. and Talatahari, S. (2010). A novel heuristic optimization method: charged system search. Acta Mech. 213: 267-289.

18. Kennedy, J. and Eberhart, R. (1995). Particle swarm optimization. Proceedings of the IEEE International Conference on Neural Networks, IEEE, Perth, Australia: 1942-1948.

19. Liu, H., Tian, H.-Q. and Li, Y.-F. (2015). Comparison of new hybrid FEEMD-MLP, FEEMD-ANFIS, Wavelet Packet-MLP and Wavelet Packet-ANFIS for wind speed predictions. Energy Conversion and Management. 89: 1-11.

20. Madhiarasan, M. and Deepa, S.N. (2016). A novel criterion to select hidden neuron numbers in improved back propagation networks for wind speed forecasting. Applied Intelligence. 44(4): 878-893.

21. Madhiarasan, M. and Deepa, S.N. (2016). Performance Investigation of Six Artificial Neural Networks for Different Time Scale Wind Speed Forecasting in Three Wind Farms of Coimbatore Region. International Journal of Innovation and Scientific Research. 23(2): 380-411.

22. Madhiarasan, M. and Deepa, S.N. (2016). Application of Ensemble Neural Networks for Different Time Scale Wind Speed Prediction. International Journal of Innovative Research in Computer and Communication Engineering. 4(5): 9610-9617.

23. Madhiarasan, M. and Deepa, S.N. (2016). ELMAN Neural Network with Modified Grey Wolf Optimizer for Enhanced Wind Speed Forecasting. Circuits and Systems. 7(10): Article in Press.

24. Mirjalili, S., Mirjalili, S.M. and Lewis, A. (2014). Grey wolf optimizer. Adv Eng Softw. 69: 46-61.

25. Mohandes, M.A., Halawani, T.O., Rehman, S. and Ahmed. A. Hussain. (2004). Support vector machines for wind speed prediction. Renewable Energy. 29: 939-947.

26. Ortiz-García, E.G., Salcedo-Sanz, S., Pérez-Bellido, A.M., Gascõn-Moreno, J., Portilla-Figueras, J.A., Prieto L. (2011). Short-term wind speed prediction in wind farms based on banks of support vector machines. Wind Energy. 14: 193-207.

27. Ramasamy, P., Chandel, S.S. and Amit Kumar Yadav. (2015). Wind speed prediction in the mountainous region of India using an artificial neural network model. Renewable Energy. 80: 338-347.

28. Rashedi, E., Nezamabadi-pour, H. and Saryazdi, S. (2009). GSA: a gravitational search algorithm. Inf. Sci. 179: 2232-2248.

29. Sander, M. Bohte., Joost, N. Kok. And Han La Poutre. (2002). Error-back propagation in temporally encoded networks of spiking neurons. Neurocomputing. 48: 17-37.

30. Sangita, B. Patil. and Bapu, K. Patil. (2015). Support Vector Machine for Wind Speed Prediction. International Journal of Research in Science \& Technology. 2: 19-25.

31. Tarek, H. M. El-Fouly., Ehab, F. El-Saadany. and Magdy, M. A. Salama. (2008). One Day Ahead Prediction of Wind Speed and Direction. IEEE Transactions on Energy Conversion. 23: 191-201.

32. Thanasis, G. Barbounis., John, B. Theocharis., Minas, C. Alexiadis. and Petros, S. Dokopoulos. (2006). Long-Term Wind Speed and Power Forecasting Using Local Recurrent Neural Network Models. IEEE Transactions on Energy Conversion. 21: 273-284.

33. Velo, R., López, P. and Maseda, F. (2014). Wind speed estimation using multilayer perceptron. Energy Conversion and Management. 81: 1-9. 
34. Wang, F., Tan, Z., Liu, D. and Qian, X. (2012). Study on wind speed prediction based on rbf neural network. Applied Mechanics and Materials. 236-237: 741-746.

35. Wang, J., Qin, S., Zhou, Q. and Jiang, H. (2015). Medium-term wind speeds forecasting utilizing hybrid models for three different sites in Xinjiang, China, Renewable Energy. 76: 91-101.

36. Xinxin Zhu., Marc, G. Genton., Yingzhong Gu. and Le Xie. (2014). Space-time wind speed forecasting for improved power system dispatch. TEST. 23: 1-25.

37. Yang, X.-S. and Deb, S. (2009). Cuckoo Search via L'evy flights. Proceedings of the World Congress on Nature \& Biologically Inspired Computing (NaBIC '09) IEEE, Coimbatore, India: 210-214.

38. Yaochu Jin., Ruojing Wen. and Bernhard Sendhoff. (2007). Evolutionary Multi-objective Optimization of Spiking Neural Networks. Artificial Neural Networks - ICANN 2007, Lecture Notes in Computer Science. 4668: 370-379.

39. Yin, D.-Y., Sheng, Y.-F., Jiang, M.-J., Li, Y.-S. and Xie, Q.-T. (2014). Short-term wind speed forecasting using Elman neural network based on rough set theory and principal components analysis. Dianli Xitong Baohu yu Kongzhi/Power System Protection and Control. 42: 46-51. 